\title{
XXXVIII. Ueber eine merkwürdige Rinnenbildung und ein neues Zwillingsgesetz an Krystallen des Andreasberger Rothgültigerzes.
}

\author{
Von \\ F. Klockmann in Aachen.
}

(Hierzu Taf. X.)

Im Jahre 1893 wurden in St. Andreasberg eine, Anzahl Pyrargyritstufen - anscheinend vom Neufanger hangenden Gang - gefunden, deren

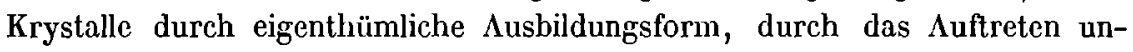
gewöhnlicher Flächen und durch eine bisher unbekannte Zwillingsverwachsung sich wesentlich von allen früheren Vorkommnissen nicht nur Andreasbergs, sondern auch der sonstigen Fundorte unterscheiden. Bei der über mehr als ein Jahrhundert erfolgten Aufspeicherung von Andreasberger Rothgültigkrystallen in zahlreichen Sammlungen und bei der intensiven Durchforschung gerade des Pyrargyrits, wovon die umfangrciche Literatur Zeugniss ablegt, muss es Wunder nehmen, dass analoge Krystalle früher nicht beobachtet worden sind. Sie scheinen auch nicht übersehen worden $\mathrm{zu}$ sein, sondern es muss sich thatsächlich um einen ganz neuen Fund handeln, denn die reichen Sammlungen der Clausthaler Bergakademie und ebenso der Andreasberger Grubenverwaltung enthalten aus früherer Zeit nichts davon. Auch Luedecke crwähnt in seinen Mineralien des Harzes von 1896 nichts Aehnliches. Bei einem gelegentlichen Besuche von St. Andreasberg habe ich wohl als der Erste die unten beschriebenen Eigenthümlichkeiten wahrgenommen und einige bessere Stufen für die Clausthaler Sammlung erworben. Andere Krystalle sind seitdem in den Handel gekommen und verstreut, doch ist mir bisher keine Schilderung derselben in der Literatur aufgestossen.

Der nachfolgenden I)arstellung liegen zwei ausgezeichnete Stufen aus 
der Clausthaler Bergakademic-Sammlung zu Grunde, für deren Herleihung ich meinem Amtsnachfolger, IIerrn Prof. Bergeat, besten Dank weiss. Dje übrigen Krystalle, soweit ich solche zu Gesicht bekommen habe, weisen die gleichen Yerhältnisse auf, mu sind sic weniger gross und weniger schün ausgebjildet. Bei der Beschreibung der einzelnen liormen halte ich absichtlich, um den Vergleich mit älteren Arlueiten zu erleichtern, an dem rhomboëdrischen Krystallsysteme des Rotlggïltigerzes fest und spreche von Rhomboëdern und Skalenoïdern, wo es richtiger trigonale und ditrigonale Pyramiden heissen müsste.

§1. Allgemeines. - Jie auf ciner Unterlage von trübem, drusigem Kalkspalh aufsitzenden P'yra.gyrilkrystalle bilden die freien Enden derben lirzes und zeigen in diesen freien Enden an allen Individuen, die immer zı Vierlingen verbunden sind, so ziemlich dieselbe Intwickelung. Die untere Krystallhälfte versehwindet in der Verwachsung, so dass dic IIemimorphie auch nicht andeutungsweise zum Ausdruck kommt. Sie sind von wechselnder Grösse, von 12 bis zu inchreren Centimetern im Durchmesser, und erreichen in ilıren Zwillingsgruppen fast Faustgrösse. Das Aussehen der Krystalle ist wie das des derben Erzes durchweg metallisch stahlgrau und stark glïnzend, wohl in Folge der Frische; bei lïngerem Liegen dürften sie dunkelgrau werden. Die kleineren Krystalle künnen zum Theil ausgesprochen rüthlich erscheinen, womit sie der $\star \Delta$ Andrensberger lichten « Varietät angehören würden, obwohl sie sich chemisch nicht von der dunkleren Abarl unterscheiden. Bemerkenswerth ist es noch, dass cinzelne Krystalle mit winzigen Kürnchen und Spitzen von Silberglinz besetzt sind, die zu der sonst schon vorhandenen Rauhigkeit vieler Flächen noch weiter beitragen.

$\S$ 2. Der Habitus ist bei allen Krystallen annähernd gleich, stumpf rhomboëdrisch, und wird bedingt durch das Vorherrschen des ersten stumpferen Rhomboëders $e=-\frac{1}{2} R\{01 \overline{1} 2\}$, dem gegenüber die seitlichen Begrenzungsflächen, das Prisma zweiter Art $a=\infty P 2\{11 \overline{2} 0\}$ und das spitze Skalenuëder $q^{\prime}=-\frac{4}{3} . R 3\{4.8 . \bar{T} \overline{2} .5\}$, gewöhnlich zurücktreten. Doch kann durch eine gelegentlich grüssere Entwickclung dieses Skalenoëders der llabitus entsprechend skalenoëdrisch beeinflusst werden. Andere Ausbildungsformen, wie die sonst am St. Andreasberger Rothgültigerz so gemeine prismatische nach $\infty P 2\{11 \overline{2} 0\}$ oder skalenoëdrische nach $R 3\{21 \overline{3} 1\}$ bezw. $R 5\{3251\}$ kommen an den mir vorliegenden Stufen nicht vor, auch fehlt es an anderen $Z$ willingsverwachsungen, als die nach dem weiter unten beschriebenen neuen Gesetz; solche Zwillinge oder richtiger Vierlinge bilden aber die Regel, Einzelkrystalle scheinen kaum vorhanden zu sein. Der Habitus der Einzelkrystalle ist in Fig. 1, Taf. $\mathrm{X}$ zum Ausdruck gebracht.

Die auffialligste lirscheinung aber, die nun sofort die vorliegenden 
Krystalle als etwas Besonderes crkennen lässt, besteht in der Ausbildung der die Polkante von $-\frac{1}{2} R\{01 \overline{1} 2\}$ gerade abstumpfenden Rhomboëderflächen ${ }_{4}^{1} R\{10 T 4\}$. Die letzteren sind in kcincm einzigen Falle einheitlich und zusammenhïngend, sondern werden in ihrer Längsrichtung, d. h. parallel der Polkante von $-\frac{1}{2} R$, durch eine deutlich ausgeprägte, hald geradlinige, bald gewundene Nedianfurche in zwei IIälften getheilt. Am Grunde der Furche erscheint dann die Jolkante von - $\frac{1}{2} R$ sichthar, und es macht den Eindruck, als wenn eine Krystallschale mit der Fläche $1 R$ einen Krystallkern ohne dicse Fläche überkruste und hindurchblicken lasse.

l)a, wo die Fläche $\frac{1}{4} R$ glatt und mit scharfen Combinationskanten ausgebildet ist, verlïuft auch die Rinne völlig geradlinig und genau in der Mittc. Sie fällt alsdann auch scnkrecht auf den inneren Kern herab, d. h. ihre seitliche Begrenzung wird durch Combination mit $a=\infty P 2\{1 / \overline{2} 0\}$ hervorgebracht. Ist dagegen, wie gewöhnlich, die von ibr getheiltc Fläche stark gestreift, so verläuft sic in wellig gebogener oder absetzender Linie und in gleicher Weise ändern sich alsdann auch die Combinationskanten der bciden lihomboëder $-\frac{1}{2} R$ und $\frac{1}{4} R$ ab.

Dic Furchenweite ist verschieden und bei gewundenem Laufe verïnderlich. Zuweilen schliesst sie sich fast ganz zusammen und erscheint, durch eine winkelig zusammenstossende Flächenstreifung deutlich markirt, eher wic einc Zwillingsnaht. In den weitaus häufigeren Fällen klafít sie auf $1-3 \mathrm{~mm}$ und selbst darüber, während ihre Tiefe sich auf $1-2 \mathrm{~mm}$ beschränkt. Von dem Aussehen der Furchen geben die Figuren 2 und $\mathbf{3}$ weiteren $\Lambda$ ufschluss.

$\S 3$. Formen und Combinationen, Flächenausbildung. Die Combinationen zeigen in Folge des Auftretens fast immer der gleichen Flächen wenig Abweichungen. Dafür hieten aber dic Flächen selbst durch dic Seltenheit ihres sonstigen Vorkommens und durch ihre Ausbildung mancherlei Bemerkenswerthes. Die beobachtelen Flächen sind die folgenden :

1. $a=\infty P^{2}\{11 \overline{2} 0\}$. Diese sonst gemeinste Form am $\Lambda$ ndreasberger Pyrargyrit fehlt auch hicr an keinem Krystalle, doch gelangen dic Flächen bei dem rhomboëdrischen ITabitus zu keiner vorherrschenden Entwickelung. Auch sind sie niemals vollzühlig vorhanden, vielmehr werden sie nicht allein durch die Verwachsung mit anderen Individuen, sondern auch durch das bevorzugte Auftreten anderer Flächen, namentlich des Skalenoëders $q^{\prime}=-\frac{s}{5} R\{4.8 .1 \overline{2} .5\}$ theilweise unterdrückt oder doch in ihren Dimensionen sehr ungleich. Glücklicherweise ist es dann eine charakteristische Streifung, die zu der nicht immer leichten Auffindung des fraglichen Prismas führt. Diese an sich etwas unregelmässige Streifung verläuft meist parallel der Combinationskante mit dem Skalenö̈der, doch kommen auch andere 
hakenförmige Streifensysteme vor nach Art der in den Lehrbüchern üblichen Zeichnung.

2. $e=-\frac{1}{2} R(0172\}$ ist die vorherrschende und keinem Krystalle fehlende Form; sie ist am oberen Pol stets vollzählig und auch annähernd symmetrisch entwickelt. Vor allen anderen Formen zeichnen sich ihre Flächen durch rauhe unebene Beschaffenheit aus, die bald durch flache parallel ihrer Symmetrielinie verlaufende Knickungen, häufiger durch unregelmässig dreieckige Gruben und Absätze hervorgebracht werden.

3. $u=\frac{1}{4} R\left\{100^{4}\right\}$, als gerade Abstumpfung der Polkanten von $-\frac{1}{2} R$, ist an allen Krystallen und auch immer vollzählig vorhanden. Diese auch sonst an Andreasberger Pyrargyriten gemeine Form ist in der auffälligsten Weise durch zwei Eigenthümlichkeiten charakterisirt: einmal durch die in \$ 2 beschriebene, niemals ganz unterdrückte Rinnenbildung und dann durch eine fiederartige Streifung parallel ihren Combinationskanten mit $W^{\prime}=$ $-\frac{1}{8} R\{0118\}$. Zuweilen kann die Streifung fehlen oder doch nur eben angedeutet sein, zumeist ist sie jedoch grob, ja treppenartig absetzend. Die Axe der Fiederstreifen bildet die genannte Längsfurche. Vergl. Fig. 2, 3 und 7 .

4. $W^{\prime}=-\frac{1}{8} R\{0118\}$ als gerade Abstumpfung der Polkanten von $\frac{1}{4} R$. Diese Form ist für den Andreasberger Pyrargyrit neu. Sie wird zwar von Sella in seinem mir leider nicht zugänglich gewesenen »Quadro delle forme cristalline dell' Argento rosso etc. 1856*, anscheinend ohne Angabe des Fundortes, aufgeführt und demzufolge von Rethwisch ${ }^{1}$ ) als gesicherte Form genannt, aber Miers ${ }^{2}$ ) versieht sie schon bei seiner Uebersicht der Formen mit einem Fragezeichen, und Luedecke ${ }^{3}$ ) erwähnt sie nicht mehr bei der Aufzählung der an Andreasberger Pyrargyritkrystallen wahrgenommenen Krystallflächen.

Bei der Grösse und für die Messung ungeeigneten Beschaffenheit der vorliegenden Krystalle lässt sich die genannte Fläche nun zwar nicht durch das Goniometer nachweisen, aber sie folgt mit aller Sicherheit direct aus der Deduction, indem sie als gerade Abstumpfung von $\frac{1}{t} R$ auftritt; ausserdem wird sie indirect durch das in $\S \&$ beschriebene Zwillingsgesetz bestätigt. Sie bildet die obere Polbegrenzung der Krystalle; gewöhnlich ist sie nur schmal entwickelt, was damit zusammenhängt, dass ihre Streifung

1) E. Rethwisch, Beiträge zur mineralogischen und chemischen Kenntniss des Rotbgültigerzes. Inaug.-Dissertation. Stuttgart 1885. Ref. diese Zeitschr. 12, 67.

2) H. A. Miers, Beiträge zur Kenntniss des Pyrargyrits und Proustits. Diese Zeitschr. 1889, 15, 129.

3) O. Luedecke, Die Minerale des Harzes. Berlin 1896. Ref. diese Zeitschr. 29, 174 . 
Ueb. eine merkwürdige Rinnenbild. u. ein neues Zwillingsgesetz an Kryst. etc. 583

parallel derjenigen auf $\frac{1}{4} R$ verläuft und somit ein Oscilliren zwischen $-\frac{1}{8} R$ und $\frac{1}{4} R$ erfolgt.

5. $q^{\prime}=-\frac{4}{5}\left\{\begin{array}{l}4.8 .1 \overline{2} .5 \\ \text {. }\end{array}\right.$ noëder, dessen Flächen stellenweise in beträchtlicher Ausdehnung, zum wenigsten aber an jedem Krystalle angedeutet vorkommen, ist für den Andreasberger Pyrargyrit bemerkenswerth. Sie wird von I,évy ${ }^{1}$ ) als an drei Andreasberger Krystallen der IIeuland'schen Sammlung auftretend zum ersten Male erwähnt, daher ihre Berücksichtigung bei Rethwisch und bei Luedecke, welch' Letzterer sie bei den isolirten, d. h. ausser Zonenverband stehenden Flächen aufführt. Dagegen bezweifeln Miers ${ }^{2}$ ) und nach ihm Goldschmidt ${ }^{3}$ ) ihre Existenz bezw. halten sie für bestätigungsbedürftig, weil die Angahe ihres Auftretens allein auf der Autorschaft von I év y beruhe.

Diese angezweifelte Form bildet nun, wie schon oben bemerkt, das am meisten hervortretende Skalenoëder der vorliegenden Krystalle. Mit Hülfe des Anlegegoniometers liessen sich an einem Krystalle, bei dem die in gerader Kante zusammenstossenden Flächen mehr als Quadratcentimeter-Grösse hatten und dabei verhältnissmässig eben waren, die Winkel der stumpfen und scharfen Polkante mit $36^{\circ}$ bezw. 720 $^{\circ}$ messen, während an einem kleineren Krystalle wenigstens der Winkel der stumpferen Kante am Reflexionsgoniometer mit aller Schärfe messbar war und sich zwischen $33^{0} \mathbf{2} 21^{\prime}$ und $33^{\prime} 10^{\prime}$ liegend ergab. Rethwisch, dem wir in der citirten Arbeit eine weitgehende Berechnung der Krystallwinkel verdanken, giebt die entsprechenden Winkel des qu. Skalenoëders zu $33^{\circ} 45^{\prime} 43^{\prime \prime}$ und $71^{\circ} 0^{\prime} 34^{\prime \prime}$ an.

Nach ihrer Ausbildung gehören die Flächen dieses steilen Skalenoëders zu den bestentwickelten; an kleineren Krystallen wird eine feine Streifung nur durch die Repetition des Goniometersignals erkennbar; auf grösseren Flächen finden sich kleine pfeilspitzenartige Unebenheiten, die durch das spitzwinkelige Zusammenstossen zweier undeutlicher Strichsysteme hervorgerufen werden.

Die genannten fünf Formen bilden anscheinend die ständige Combination eines jeden Krystalles; sie sind die wesentlichen, wenn auch in manchen Fällen nicht die einzigen Formen. Was sich aber sonst noch gelegentlich an Flächen einstellt, gehört Skalenoëdern an. Zunächst ist zu bemerken, dass in einem Falle an Stelle des gewöhnlichen Skalenoëders $q^{\prime}$ ein ähnliches negatives auftritt, dessen Flächen in der Zone $\left[-\frac{1}{2} R . \infty P 2\right]$ liegen und dessen stumpfer Polkantenwinkel mittelst des

1) Lévy, Description d'une collection de minéraux, formée par M. Heuland. Londres 1837, 2.

2) Miers, l. c.

3) V. Goldschmidt, Index der Krystallormen. Berlin 1891, 3. 
Anlegegoniometers zu $39^{\circ}$ gemessen werden konnte. Unter den sonst von St. Andreasberg angegehenen Skalenoëdern finde ich keine Form, die darauf passt.

Häufiger sind die Rhomboëderflächen von $c=-\frac{1}{2} R\{01$ T2\} parallel ihrer Mittellinie flach geknickt, was auf ein negatives Skalenoëder der Zone $\left[-\frac{1}{2} R . R\right]$ hinweist. Dieses llache Skalenoëder tritt entweder geradezu als Ersatz für das Rhomboëder $e$ auf oder beide Formen treten neben einander in Combination. Eine Winkel-, bezw. Indicesbestimmung des fraglichen Skalenoëders war nicht ausführbar, doch finden sich nach Luedecke an Andreasberger Krystallen drei negative Skalenoëder in der genannten Zone, von denen $-\frac{1}{5} R 3\{12 \overline{3} 5\}$ den stumpfsten Polkantenwinkel mit $16^{\circ} 18^{\prime}$ besitzt und der fraglichen Form wohl entsprechen könnte.

§ 4. Zwillinge. Die vorstehend geschilderten Krystalle treten nun ausnahmslos zu Zwillingen, richtiger zu Vierlingen zusammen, und zwar nach einem bisher nicht beobachteten Zwillingsgesetze. Nach der Zusammenstellung von Miers $\left.{ }^{1}\right)$ sind am Pyrargyrit folgende Gesetze bekannt:

$$
\begin{aligned}
& \text { 1) nach } u=\frac{1}{4} R\{10 \overline{4}\} \text {, } \\
& \text { 2) }-r=R\{10 \bar{T}\} \text {, } \\
& 3)-a=\infty P 2\{11 \overline{2} 0\}, \\
& \text { 4) }-e=-\frac{1}{2} R\{01 T 2\} .
\end{aligned}
$$

Rethwisch und Schuster ${ }^{2}$ ) lassen dann auf Grund alterer Angaben noch die beiden weiteren Gesetze nach $o=0 R\{0001\}$ und $b=\infty R\{10 \overline{10}\}$ $\mathrm{zu}$, welche jedoch von Miers mit Recht auf $a=\infty P 2\{11 \overline{2} 0\}$ zurückgeführt werden. Nach Frenzel $\left.{ }^{3}\right)$ sollen weiter noch Zwillinge nach $s=$ $-2 R\{02 \overline{2} 1\}$ vorkommen, was aber von Weisbach, Rethwisch $\left.{ }^{4}\right)$ und Miers angezweifelt bezw. nicht anerkannt wird. Somit ist die nachstehend erörterte $Z$ willingsverwachsung das fünfte sichere Gesetz am Pyrargyrit.

Die Zwillingslläche dieses neuen Gesetzes giebt die oben näher beschriebene Fläche $W^{\prime}=-\frac{1}{8} R\{01 \overline{1} 8\}$ ab, die Verwachsungsfläche wird von einer dazu senkrechten Ebcne geliefert.

Grösse und sonstige Beschaffenheit der mir vorliegenden Krystalle sind der Feststellung dieses Zwillingsgesetzes durch Messung nicht besonders günstig, aber schon die üussere Betrachtung genügt zur sicheren und unanfechtbaren Bestätigung. Die zu einem Zwillinge miteinander verbundenen Individuen sind annähernd von gleicher Grösse und verleihen demselben dadurch ein ziemlich symmetrisches Ansehen. In Folge dessen

1) Miers, l. c. S. $162 \mathrm{ff}$.

2) Schuster, Ueber hemimorphe Pyrargyritzwillinge von Andreasberg. Diese Zeitschr. 1887, 12, $117 \mathrm{f}$.

3) Frenzel, cf. Rethwisch, l. c. S. 83.

4) Rethwisch, l. c. S. 83; cf. auch Goldschmidt, Index 3, 66. 
liegen auch diejenigen Flächen, die boiden Individuen gemeinsam sind, also die Zwillingsflächen $W^{\prime}$, in gleicher Ilöhe und in gegenseitiger Fortsetzung; sic spiegeln gleichzeilig ein. Das zeigt sich in augenfälliger Weise und wird noch dadurch besonders auffällig, weil aurh hei geeignetem Reflexionswinkel gleichzeitig die treppenartige Streifung von $\frac{1}{4}_{R} R$, die ja von der sich stetig wiederholenten Combination mil $-\frac{1}{8} l$, borührt, cbenfalls rinspiegelt.

Dancben habe, ich versuchi, das Geselz durch Messung zu erhärten. Von dem einspringenden Winkel $o: \underline{e}$ wurdc cin Wachsabdruck gemacht und dessen Seiten mil Spiegelglas belegt. Der mit dem Anlegegoniometer gemessene Winkel ergab an mehreren Krystallen im Jurchschnitt 39", während er unter Zugrundelegung der Winkeltabelle von Rethwisch $390179^{\prime}$ betragen sollte.

Aus der weiteren Rechnung folgt, dass sich dic Verticalaxen beider Individuen unter einem Winkel von ca. $13^{0}$ schneiden und dass die auf der '/willingsfläche senkrecht stehende Verwachsungsfläche - zumeist geschicht allerdings dic Verwachsung in unregelmässiger Fläche - ungefähr einem positiven Rhomboëder $9,7 R$ entsprechen würde.

Das besprochene Zwillingsgesetz tritt nun in allen beobachteten Fällen in symmetrischer Wiederholung nach allen drei Rhomboëderflächen von $W^{\prime}=-\frac{1}{8} R\{01 T 8\}$ auf, so dass cyklische Vicrlinge entstehen, die in ihrer symmetrischen Gruppirung an dic bouquctartigen Vierlinge des Pyrargyrits nach $u=\frac{1}{4} R\{10 T 4\}$ erinnern, aber davon doch wiederum ganz verschicden sind. Durch die Gegenüberstellung beider Vierlingsarten in der schematischen Zeichnung (Fig. 4 und 5) tritt der Unterschied sofort in die Augen. Die Fig. 6 und 7 geben die Vierlinge in ihrer gewöhnlichen Combination wieder; bei diesen idealisirten Bildern treten aber dic Flächen des Prismas zweiter Art über Gebühr hervor, was in der Natur nicht der Fall ist. Immerhin besitzen die natürlichen Krystalle einen hohen Grad symmetrischen Aussehens, der nur durch gelegentliche parallele Wiederholungen einzelner Kanten etwas beeinträchtigt wird.

Jie vorstehenden Betrachtungen lehren, dass die so viel durchforschten $\Lambda$ ndreasberger Rothgültigkrystalle immer noch neuc Ergebnisse zu licfern vermögen. Eine erschöpfende Monographic dieses Harzer Minerals wird wohI noch einige Zeit ungeschrieben bleiben müssen.

Aachen, den 18. März 1900. 


\section{Erläuterung der Figuren.}

Fig. 1. Gewöhnlicher Habitus der Krystalle.

$$
\begin{aligned}
& a=\infty P 2\{11 \overline{2} 0\} \\
& q=-\frac{5}{5} R 3\{4.8 . \overline{1} 2.5\} \\
& e=-\frac{1}{2} R\{01 \overline{1} 2\} \\
& u=-\frac{1}{4} R\{10 \overline{1} 4\} \\
& W^{\prime}=-\frac{1}{8} R\left\{01^{1} 8\right\} .
\end{aligned}
$$

Fig. 2. Krystall mit Rinne und Streifung auf der Fläche $u=\frac{1}{4} R\{10 \overline{4}\}$ und charakteristischer Zeichnung der übrigen Flächen.

Fig. $3 \mathbf{a}$ und $3 \mathbf{b}$. Specielle Darstellung der Rinnen.

Fig. 4. Schematische Darstellung eines Vierlings nach dem Gesetz: Zwillingsfläche $W^{r}=-\frac{1}{8} R\{01 \overline{1} 8\}$.

Fig. 5. Zum Vergleich: schematische Darstellung eines Vierlings nach dem Gesetz: Zwillingsfläche $u=\frac{1}{4} R\{10 \overline{4} 4\}$.

Fig. 6. Vierling nach $W^{\prime}=-\frac{1}{8} R$ mit allen auftretenden Flächen.

Fig. 7. Vierling nach $W^{\prime}=-\frac{1}{8} R$ mit Rinnen und Streifung auf den Flächen $u=\frac{1}{4} R\{10 \overline{4} 4\}$. 